\title{
Protocolo Unificado de Plastinación con Silicona en Frío y a Temperatura Ambiente
}

\author{
Unified Plastination Protocol with Silicone at Cold and Room Temperature
}

\begin{abstract}
Nicolás E. Ottone $e^{1,2,3,4}$
OTTONE, N. E. Protocolo unificado de plastinación con silicona en frío y a temperatura ambiente. Int. J. Morphol., 39(2):630-634, 2021.

RESUMEN: En la actualidad, la técnica de plastinación es considerada una de las más novedosas formas de conservación cuerpos completos, secciones y órganos, tanto humanos como animales, para su uso en docencia de pre y postgrado, como así también investigación morfológica. En este sentido, para desarrollar las diversas técnicas de plastinación se requiere de equipamiento específico y formación especializada de académicos, que tengan la capacidad de llevar adelante la diversidad de protocolos que existen, según el material anatómico que se desee preservar. En el año 2015, desde el Laboratorio de Plastinación y Técnicas Anatómicas de la Universidad de La Frontera, se propuso por primera una nueva técnica de plastinación a temperatura ambiente, que permitió obtener preparaciones plastinadas de igual calidad que las técnicas clásicas de plastinación. En la actualidad, desde nuestro laboratorio, se propone un nuevo protocolo de plastinación con silicona que unifica las técnicas que se desarrollan en frío como así también a temperatura ambiente para la conservación de cuerpos humanos y animales completos, secciones anatómicas, regiones corporales, y órganos aislados.
\end{abstract}

PALABRAS CLAVE: Plastinación en frío; Plastinación a temperatura ambiente; Silicona; Anatomía humana; Anatomía veterinaria.

\section{INTRODUCCIÓN}

La plastinación es una técnica anatómica utilizada para preservar microscópicamente el material biológico. Fue desarrollada por el Dr. Gunther von Hagens en Heidelberg, Alemania en 1977 (von Hagens 1979, 1986; von Hagens et al., 1987; Ottone, 2013; Ottone et al., 2015; Ottone, 2018). Este método preserva el material cadavérico, tal como especímenes biológicos, y particularmente material blando tal como cerebros, corazones, riñones, pulmones, hígados y músculos, en el campo de la anatomía y patología humana y animal. En este proceso, el agua y la grasa en los tejidos biológicos se sustituyen por polímeros plásticos, tales como resinas de silicona, epoxi o poliéster (von Hagens et al., 1987; Baptista et al., 1988). Posteriormente, estos materiales se vuelven duros, lo que resulta en especímenes secos, inodoros y altamente duraderos (von Hagens et al., 1987; Ottone et al., 2015). El tipo de polímero utilizado determina las propiedades visuales [epoxi: transparentes (Ottone et al., 2018a,b), poliéster: opacas (Guerrero et al., 2019)] y el posible movimiento (flexible o firme) del espécimen embebido (Ottone et al., 2014). Una vez incrustado el espécimen, es mucho más estable que los especímenes congelados, deshidratados o encerados con parafina. Además, esta técnica tiene una gran ventaja: conserva la topografía superficial original, incluso la identidad celular a nivel microscópico (deJong \& Henry, 2007; Ottone et al., 2016; Prieto et al., 2019; Ottone et al., 2020).

La plastinación es la técnica anatómica más novedosa que permite conservar los tejidos biológicos perecederos (órganos aislados, cuerpos humanos y animales completos, secciones anatómicas y regiones corporales aisladas, como así también secciones ultrafinas de material biológico). Los tejidos se vuelven secos y no perecederos mediante el uso de diferentes polímeros y plásticos especiales (Raoof et al., 2007; Ottone et al., 2015).

\footnotetext{
${ }^{1}$ Laboratorio de Plastinación y Técnicas Anatómicas, Centro de Investigaciones en Ciencias Odontológicas (CICO), Facultad de Odontología, Universidad de La Frontera, Temuco, Chile.

${ }^{2}$ Departamento de Odontología Integral del Adulto, Facultad de Odontología, Universidad de La Frontera, Temuco, Chile.

${ }^{3}$ Centro de Excelencia en Estudios Morfológicos y Quirúrgicos (CEMyQ), Facultad de Medicina, Universidad de La Frontera, Temuco, Chile.

${ }^{4}$ Programa de Doctorado en Ciencias Morfológicas, Facultad de Medicina, Universidad de La Frontera, Temuco, Chile.
} 
La plastinación es una técnica ampliamente aceptada debido a la durabilidad y al alto valor de la investigación ofrecidos por tales especímenes (Bickley et al., 1981). La plastinación es un método de preservación de tejidos que ha demostrado su capacidad de aplicación en diferentes campos de la docencia e investigación en ciencias morfológicas, anatomía normal y patológica. Con la técnica de plastinación se logra preservar el material cadavérico, sin la toxicidad del formaldehído, y además se obtienen preparaciones disecadas de alta calidad que conservan su tamaño y forma, en un corto período de tiempo y a bajo costo. Esto es beneficioso en momentos en que los cadáveres humanos para la investigación son escasos y difíciles de obtener. Además, la técnica de plastinación logra la preservación de los tejidos seccionados, constituyéndose en una excelente herramienta de enseñanza e investigación (Dawson et al., 1990; Sora et al., 2012; Ottone et al., 2014, 2015). Una aplicación novedosa, y también única hasta el momento, consistió en la extracción de ADN intacto de muestras plastinadas, a partir de la técnica de plastinación a temperatura ambiente desarrollada en nuestro laboratorio (Ottone et al., 2020). De esta manera, se comprueba la conservación de las características del tejido plastinados, pudiendose aplicar este conocimiento para la investigación en diversos ámbitos, como las ciencias forenses. Además, los especímenes secos e inodoros, con facilidad en su manipulación y mantención, se convierten en herramientas útiles para enseñar la anatomía desde un punto de vista macro y microscópico.

En 2015, nuestro grupo de investigación publicó por primera vez (Ottone et al., 2015) una propuesta de plastinación con silicona a temperatura ambiente con varias novedades que modificaban la técnica tradicional de plastinación a temperatura ambiente desarrollada por Zheng et al. (1998) y Glover et al. (1998), y que incorporaba principios de la técnica de plastinación con silicona en frío desarrollada por Gunther von Hagens. En este sentido, las modificaciones y propuestas sugeridas en este nuevo protocolo estuvieron asociadas al paso de impregnación forzada, en la cual se describieron por primera vez los conceptos de impregnación forzada activa y pasiva, asociados al encendido y apagado de la bomba de vacío, respectivamente, durante el proceso de impregnación forzada. Además, la mezcla silicona:catalizador de esta etapa fue similar a la utilizada en la técnica de plastinación en frío (von Hagens 1979, 1986). Lo mismo en la etapa de curado, se utilizó el compuesto TEOS para gasificar las muestras y acelerar el proceso de secado y endurecimiento de las muestras, a diferencia de lo que se proponía clásicamente en la técnica de plastinación a temperatura ambiente. Recientemente, también a partir de investigaciones desarrolladas en nuestro laboratorio, definimos el concepto de microplastinación, uniendo la plastinación con el desarrollo de protocolos ex- perimentales en animales, para la visualización de los cambios microanatómicos ocasionados por patologías de interés en estructuras anatómicas de relevancia para la salud de las personas (Ottone, 2020; Vargas et al., 2020).

En este artículo, se propone unificar los protocolos de plastinación en frío y a temperatura ambiente de Ottone et al. (2015) bajo un mismo protocolo, identificando diferencias únicamente en el paso de la impregnación forzada, y asociadas a la cámara de vacío y los tiempos de impregnación. A continuación se detallará el nuevo protocolo unificado de plastinación con silicona.

\section{Protocolo Unificado de Plastinación con Silicona}

\section{A. Deshidratación}

Este paso es igual tanto para la técnica de plastinación en frío como a temperatura ambiente. Como la plastinación consiste en reemplazar el agua de los tejidos por un polímero del tipo silicona, epoxi o poliéster, y debido a que éstos polímeros no son miscibles en agua, es necesario utilizar un componente intermediario, un disolvente orgánico, que sea miscible en agua y en el polímero. Es por eso que se recurre a la acetona. Para reducir la retracción que ocasiona, se realiza la sustitución por congelación (en frío: $-25^{\circ} \mathrm{C}$ ) de los líquidos que presente la muestra biológica por acetona. En caso que las muestras biológicas estén conservadas con otros compuestos distintos a la formalina, como glicerina, previo a la acetona será necesario sumergir las muestras en etanol al $50 \%$ durante al menos 1 semana. En caso de contener etanol, o mismo formalina, previo a la deshidratación será necesario lavar los preparados en agua corriente durante varios días, dependiendo del tamaño de los preparados. La deshidratación con acetona, en comparación al etanol, cuyo proceso consiste en el pasaje de las muestras por diversas concentraciones de etanol, es mucho más sencillo de llevar adelante, además de ocasionar una menor retracción de los tejidos. Además, la acetona también es desgrasante, por lo cual permitirá avanzar también en la eliminación de la grasa de los tejidos, importante para una correcta aplicación de la técnica de plastinación.

El proceso de deshidratación, por lo tanto, consistirá en la sustitución por congelación en acetona a $-25^{\circ} \mathrm{C}$, durante un mínimo de 4 semanas. En cada semana el material biológico será traspasado de un baño de acetona al subsiguiente, en concentraciones superiores de acetona cada baño, comenzando al $90 \%$ de acetona en el primer baño, hasta alcanzar en el último baño de acetona, con un $100 \%$ de acetona. Clásicamente, se describe que la relación tejido/ acetona, debe ser 1:10. Sin embargo esta puede ser inferior. En caso de deshidratar órganos huecos, será importante lle- 
nar los mismos con acetona fría $\left(-25^{\circ} \mathrm{C}\right)$ al momento de sumergirlos en la misma, constribuyendo al mantenimiento de la forma y también a una disminución de la retracción.

\section{B. Desengrasado}

Este paso es igual tanto para la técnica de plastinación en frío como a temperatura ambiente. El desengrasado consiste en eliminar la grasa de las muestras biológicas. Ésta etapa comienza durante la deshidratación. En el caso de la plastinación con silicona, y de muestras de gran tamaño, finalizado el proceso deshidratación, se trasladarán las muestras biológicas a contenedores con acetona al $100 \%$, a temperatura ambiente. La acetona a temperatura ambiente presenta una mayor potencia desgrasante, favoreciendo la eliminación de grasa de los tejidos. Esta etapa tiene una duración de algunos días a semanas, dependiendo del tamaño de la muestra. Sin embargo, el mejor compuesto desengrasante es el cloruro de metileno, el cual se reserva para la plastinación de cortes con resina epoxy, en la cual se requiere lograr una gran transparentación de los tejidos. En el caso de la plastinación con silicona, no se justifica su uso, debido a la gran cantidad que debería usarse fundamentalmente asociado a su elevada toxicidad, siendo peligroso para la salud, por lo cual se reserva su utilización en la plastinación de cortes con resina epoxy.

\section{Impregnación Forzada}

El material biológico, totalmente embebido en acetona por los pasos previos, será sumergido en una mezcla de polímero de silicona:catalizador (silicona: polidimetilsiloxano + catalizador: dilaurato de dibutilestaño / proporciones: 100:1), que tienen una presión de vapor baja (alto punto de ebullición). Al mezclar el polidimetilsiloxano (monómero de silicona) con el dilaurato de dibutilestaño (catalizador, alargador de cadena) se inicia la formación de moléculas de silicona de cadena larga, produciendo un fluido de alta viscosidad, siendo éstas moléculas de cadena larga las que son incorporadas dentro del material biológico durante el proceso de impregnación forzada. El proceso de impregnación forzada consistirá en la extracción de la acetona (disolvente intermediario) del interior del material biológico y su reemplazo por la mezcla de polímero:catalizador, mediante la aplicación de una presión de vacío creciente. El intermediario volátil (acetona) que se encuentra dentro del espécimen es removido por la acción de la bomba de vacío (impregnación forzada activa). Conforme la acetona es removida, una diferencia de presión determinará que el polímero entre al espécimen. La impregnación forzada debe llevarse a cabo lentamente conforme el polímero ingresa dentro del espécimen donde la acetona cambia de estado líquido a gaseoso y es removida en forma de burbujas. Esto último ocurre desde los $240 \mathrm{mmHg}$, presión a la cual la acetona se volatiliza, y per- mite su extracción del interior de las muestras en forma de burbujas. La velocidad de impregnación es cuidadosamente ajustada por una adición controlada de aire dentro de la bomba de vacío por medio de una válvula de "bypass". La duración de la etapa de impregnación forzada dependerá, principalmente del tamaño del espécimen (y la cantidad), la densidad del tejido y la viscosidad del polímero utilizado. Durante este periodo el vacío debe ser intensificado de una presión de $760 \mathrm{mmHg}$, de acuerdo a la formación deseada de burbujas (intermedio), a una presión de, aproximadamente, $10 \mathrm{mmHg}$, donde las burbujas pequeñas irán a la superficie (la visualización de burbujas es indicativo de la salida de la acetona del interior del especimen). Una vez alcanzado este nivel final de presión, y con ausencia de burbujas (indicador del reemplazo de la acetona por la silicona), entonces la etapa de impregnación forzada ha llegado a su fin, y el espécimen es removido de la solución del polímero, luego de 24 horas de finalizada esta etapa. El proceso aquí explicado es común tanto para la plastinación en frío como a temperatura ambiente, identificándose diferencias en el tamaño y cantidad de burbujas: en frío, las burbujas son de gran tamaño y se presentan un baja cantidad (ejemplo, una burbuja por segundo); a temperatura ambiente, las burbujas son pequeñas y se presentan en gran cantidad (ejemplo, cinco burbujas por segundo).

\section{Cámara de Vacío}

Plastinación en Frío: las muestras biológicos son sumergidas en la mezcla silicona:catalizador dentro de una cámara de vacío ubicada en el interior de un refrigerador, a $-25^{\circ} \mathrm{C}$.

Plastinación a Temperatura Ambiente: el material biológico es sumergido en la mezcla de silicona:catalizador dentro de una cámara de vacío, la cual se encuentra en un ambiente a una temperatura ambiente de $20-22^{\circ} \mathrm{C}$. En este caso, la cámara de vacío no se encuentra en el interior de un freezer.

\section{Manipulación de la bomba de vacío}

La manipulación de la bomba de vacío será igual tanto para la técnica de plastinación en frío como a temperatura ambiente. En 2015 (Ottone et al., 2015), se definió por primera vez el concepto de impregnación forzada activa y pasiva, con la cual se definió que no es necesario que la bomba de vacío funcione continuamente, desde los $760 \mathrm{mmHg}$ hasta alcanzar la presión final de $10 \mathrm{mmHg}$, cómo clásicamente describió von Hagens en los inicios, y como ha sido repetido en todos los protocolos de plastinación desarrollados posteriormente. De esta manera, se pueden establecer 8 horas diarias de funcionamiento de la bomba de vacío (impregnación forzada activa) en las cuales se controla la reducción de la presión, a través de la manipulación de la válvula bypass, per- 
mitiendo de esta manera la extracción activa de la acetona del interior de las muestras, lográndose el intercambio por la mezcla silicona-catalizador. Posteriormente, se apaga la bomba de vacío, manteniendo la presión que se alcanzó finalmente (impregnación forzada pasiva), para el día posterior continuar con el proceso activo de impregnación forzada. Esto no supone una prolongación del tiempo de impregnación, asegurando de esta manera un control riguroso y más seguro del funcionamiento de las bombas de vacío, manteniéndose en funcionamiento mientras nos encontramos en el laboratorio.

\section{Tiempos de la impregnación forzada:}

Plastinación en Frío: la polimerización de la silicona comienza inmediatamente al combinarla con el catalizador. Al ser incorporada dentro de una cámara de vacío a muy bajas temperaturas $\left(-25^{\circ} \mathrm{C}\right)$, el proceso de polimerización se enlentece, pero la viscosidad de la mezcla aumenta, por lo cual, el proceso de impregnación forzada debe ser llevado adelante muy lentamente, para que el ingreso de la silicona al interior de la muestra se realice suavemente y de esta manera no exista el peligro de hacer un ingreso acelerado de la silicona, que provocaría una reducción acelerada de la presión y una finalización prematura de proceso de impregnación forzada, con impregnación incompleta de la muestra. De esta manera, el tiempo mínimo de impregnación forzada es de 1 mes, aumentando según el tamaño y cantidad de muestras. En relación a la durabilidad de la mezcla silicona:catalizador, siempre y cuando se mantenga en el interior de la cámara de vacío en frío $\left(-25^{\circ} \mathrm{C}\right)$, la silicona se mantendrá sin endureceder por un tiempo prolongado (puede ser por años), esto es porque el frío enlentece notablemente el avance del proceso de polimerización.

Plastinación a Temperatura Ambiente: la polimerización de la silicona también comienza inmediatamente al combinarla con su correspondiente catalizador. Pero a temperatura ambiente, la silicona se mantiene más fluida, al menos durante el proceso de impregnación forzada, ya que la misma, a temperatura ambiente, tiene una duración mucho más reducida (de una semana a un mes, como máximo, también dependiendo de la cantidad y tamaño de las muestras). Sin embargo, el proceso de polimerización y endurecimiento de la mezcla silicona:catalizador es mucho más rápido, y su reutilización se ve limitada por este motivo. Por eso, una recomendación para aumentar la vida útil de la mezcla silicona:catalizador, es que una vez que se finaliza la impregnación forzada, colocar inmediatamente la mezcla dentro de un freezer a $-25^{\circ} \mathrm{C}$, sin embargo, a pesar de realizar esto, la vida útil de la mezcla igualmente será mucho menos en comparación a la mezcla utilizada en el proceso de impregnación forzada en frío.

\section{Posicionamiento}

Consiste en la revisión de los distintos elementos anatómicos en el material biológico, determinando la separación de estructuras superficiales para la visualización de estructuras anatómicas más profundas, como así también la identificación de elementos vasculonerviosos, o cualquier otro elemento, además de favorecer la eliminación del exceso de silicona incorporado en el material en la etapa de impregnación forzada. Pueden utilizarse cuerdas, agujas, separadores, cualquier elemento que permita mantener las estructuras anatómicas en la posición que se requiera, siempre cuidando de no presionar excesivamente los elementos a utilizar sobre las estructuras anatómicas, para no provocar marcas sobre el tejido que luego serán imposibles de eliminar al endurecer finalmente el preparado. El posicionamiento también puede iniciarse al principio del proceso de plastinación, antes del inicio de la deshidratación.

\section{E. Curado}

Este paso es igual tanto para la técnica de plastinación en frío como a temperatura ambiente. Para el curado, se utiliza un líquido que se gasifica (TEOS, tetraetil ortosilicato) sobre muestras biológicas, dentro de una cámara hermética. El TEOS interactúa con el catalizador (dilaurato de dibutilestaño), contenido en la mezcla de impregnación, iniciando la interacción de las moléculas de silicona mediante una polimerización de extremo a extremo. De esta manera, la incorporación del TEOS (entrecruzador), provoca el entrecruzamiento y la unión de las moléculas de silicona de cadena larga (conformadas por la unión de la silicona con su catalizador), formando una estructura tridimensional. Debido a esta reticulación, la silicona dentro de la muestra se solidificará y se secará. La superficie de la muestra se cura rápidamente, pero la difusión del gas hacia interior de la muestra es más lenta. Para garantizar un curado adecuado durante todo el proceso, la muestra debe guardarse en bolsas plásticas herméticas durante el tiempo que permita su secado final.

\section{CONCLUSIONES}

A partir del protocolo unificado de plastinación con silicona, se puede desarrollar la técnica de plastinación, tanto en frío, como a temperatura ambiente, obteniendo los mismos resultados que las técnicas de plastinación tradicionales: muestras biológicas totalmente bioseguras, y secas, que mantienen las características morfológicas de manera imperecedera, sin sufrir deterioro, y permitiendo la manipulación segura de las muestras, al haberse eliminado el formaldehído, y a su vez, permitiendo mantener en forma exacta todos los elementos 
anatómicos y las relaciones topográficas que se establecen entre ellos, algo que no es posible mantener a largo plazo en los especímenes frescos o preservados con formalina, los cuales sufren un alto deterioro a lo largo del tiempo. La única diferencia a considerar es la "ubicación" de la cámara de vacío, que si es dentro de un freezer, corresponderá a la plastinación en frío, y si es fuera de un freezer, en un ambiente a $20-22{ }^{\circ} \mathrm{C}$, corresponderá a la plastinación a temperatura ambiente. Sin embargo, con el uso de este protocolo unificado de plastinación con silicona, se evita caer en estas diferencias, pudiendo aplicar este protocolo en cualquiera de las dos opciones de cámara de vacío. La diferencia que sí deberá considerarse está en relación a los tiempos de la impregnación forzada, la cual será más prolongada cuando la plastinación se realice en frío, con todas las características ya descritas anteriormente y sus diferencias con la plastinación a temperatura ambiente. De este modo, se propone un protocolo unificado de plastinación con silicona, que puede aplicarse para la preservación biosegura, y por un tiempo indefinido, de cuerpos humanos y animales completos, como así también secciones anatómicas, regiones corporales, y órganos aislados. Este protocolo unificado de plastinación con silicona podrá desarrollarse para la preservación de material biológico humano y animal destinado a la docencia de pregrado, postgrado, permitiendo la aplicación del conocimiento anatómico en la clínica, cirugía e imagenología, como así también para el desarrollo de investigación anatómica y morfológica, con aplicación en modelos experimentales animales para estudiar las modificaciones macroanatómicas y microanatómicas de diversas patologías de interés en la población.

OTTONE, N. E. Unified plastination protocol with silicone at cold and room temperature. Int. J. Morphol., 39(2):630-634, 2021.

SUMMARY: At present, plastination technique is considered one of the newest forms of conservation of whole bodies, sections and organs, both human and animal, for use in undergraduate and graduate teaching, as well as morphological research. In this sense, to develop the various plastination techniques requires specific equipment and specialized training of academics, who have the ability to carry out the diversity of protocols that exist, according to the anatomical material that is to be preserved. In 2015, from the Laboratory of Plastination and Anatomical Techniques of Universidad de La Frotera, a new plastination technique was proposed for the first time at room temperature, which allowed obtaining plastinated preparations of the same quality as the classic plastination techniques. At present, from our laboratory, a new protocol for plastination with silicone is proposed that unifies the techniques that are developed in cold as well as at room temperature for the conservation of complete human and animal bodies, such as as well as anatomical sections, body regions, and isolated organs.

KEY WORDS: Cold plastination; Room temperature plastination; Silicone; Human anatomy; Veterinary anatomy.

\section{REFERENCIAS BIBLIOGRÁFICAS}

Baptista, C. A. C.; Cerqueira, E. P. \& Conran, P. B. Impregnation of biological specimens with resins and elastomers: plastination with Biodur S10 resin. Rev. Bras. Cienc. Morfol., 5(1):60-2, 1988.

Bickley, H. C.; von Hagens, G. \& Townsend, F. M. An improved method for the preservation of teaching specimens. Arch. Pathol. Lab. Med., 105(12):674-6, 1981.

Glover, R. A.; Henry, R. W. \& Wade, R. S. Polymer Preservation Technology: POLYCUR. A Next Generation Process for Biological Specimen Preservation. Resumen. J. Int. Soc. Plastination, 13(2):39, 1998.

Guerrero, M.; Vargas, C.; Alarcón, E.; del Sol, M. \& Ottone, N. E. Desarrollo de un protocolo de plastinación de cortes con resina poliéster aplicado a secciones de cerebro humano. Int. J. Morphol., 37(4):1557-1563, 2019.

Ottone, N. E. Gunther von Hagens, Creador de la plastinación. Reseña histórica y desarrollo de la técnica. Rev. Argent. Anat. Online, 4(2):70-6, 2013.

Ottone, N. E.; Cirigliano, V.; Lewicki, M.; Bianchi, H. F.; Aja Guardiola, S.; Algieri, R. D.; Cantin, M. \& Fuentes, R. Plastination Technique in laboratory rats: An alternative resource for teaching, surgical training and research development. Int. J. Morphol., 32(4):1430- 5, 2014.

Ottone, N. E.; Cirigliano, V.; Bianchi, H. F.; Medan, C. D.; Algieri, R. D.; Borges Brum, G. \& Fuentes, R. New contributions to the development of a plastination technique at room temperature with silicone. Anat. Sci. Int., 90(2):126-35, 2015

Ottone, N. E.; del Sol, M. \& Fuentes, R. Report on a sheet plastination technique using commercial epoxy resin. Int. J. Morphol., 34(3):1039-43, 2016.

Ottone, N. E. Plastinación: Fundamentos de las técnicas y su implementación en la Universidad de La Frontera. J. Health Med. Sci., 4(4):293-302, 2018.

Ottone, N. E.; Baptista, C.; Latorre, R.; Bianchi, H. F.; del Sol, M. \& Fuentes, R. E12 sheet plastination - Techniques and applications. Clin. Anat., 31(5):742$56,2018 \mathrm{a}$

Ottone, N. E.; Vargas, C. A.; Veuthey, C.; del Sol, M. \& Fuentes, F. Epoxy sheet plastination on a rabbit head-new faster protocol with Biodur ${ }^{\circledR}$ E12/E1. Int. J. Morphol., 36(2):441-6, 2018 b.

Ottone, N. E. Micro-plastination. Technique for obtaining slices below $250 \mathrm{~mm}$ for the visualization of microanatomy in morphological and pathological experimental protocols. Int. J. Morphol., 38(2):389-391, 2020.

Ottone, N. E.; Baptista, C. A. C.; del Sol, M. \& Muñoz Ortega, M. Extraction of DNA from plastinated tissues. Forensic Sci. Int., 309:110199, 2020.

Prieto, R.; Vargas, C. A.; Veuthey, C.; Aja-Guardiola, S. \& Ottone, N. E. Conceptos fundamentales del protocolo modificado de plastinación a temperatura ambiente con silicona, con posterior pigmentación, y su aplicación para la conservación de placenta humana. Int. J. Morphol., 37(1):369-74, 2019.

Vargas, C. A.; Baptista, C. A. C.; Del Sol, M.; Sandoval, C.; Vásquez, B.; Veuthey, C. \& Ottone, N. E. Development of an ultrathin sheet plastination technique in rat humeral joints with osteoarthritis induced by monosodium iodoacetate for neovascularization study. Anat. Sci. Int., 95(2):297-303, 2020.

von Hagens, G. Impregnation of soft biological specimens with thermosetting resins and elastomers. Anat. Rec., 194(2):247- 55, 1979.

von Hagens, G. (Ed.). Heidelberg Plastination Folder. Collection of Technical Leaflets of Plastination. Heidelberg, Biodur Products GmbH, 1986.

von Hagens, G.; Tiedemann, K. \& Kriz, W. The current potential of plastination. Anat. Embryol. (Berlin), 175(4):411-21, 1987.

Zheng, T.; Liu, J. \& Zhu, K. Plastination at room temperature. J. Int. Soc. Plast., 13(2):21-5, 1998.

Dirección para correspondencia:

Dr. Nicolás Ernesto Ottone

Profesor Asociado

Laboratorio de Plastinación y Técnicas Anatómicas

Centro de Investigación en Ciencias Odontológicas (CICO)

Facultad de Odontología

Universidad de La Frontera

Temuco - CHILE

Recibido: 22-11-2020

Aceptado: 14-01-2021

E-mail: nicolas.ottone@ufrontera.cl 